\title{
Cultura y educación superior en la formación en jurisprudencia en la Uniandes, extensión Riobamba, Ecuador
}

\author{
" Culture and superior education in the formation \\ in jurisprudence in the Uniandes, extension \\ Riobamba, Ecuador
}

\begin{tabular}{l} 
(iD) Janneth Ximena Iglesias Quintana \\
iD Lola Ximena Cangas Oña \\
$\frac{\text { iD Elías Machado Maliza }}{\text { Universidad Regional Autónoma de los Andes, Ecuador }}$ \\
\hline
\end{tabular}

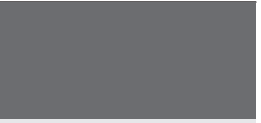

\section{Resumen}

El proceso formativo de los estudiantes de la carrera de Derecho en la extensión Riobamba de Uniandes, Ecuador, exige de la articulación de la cultura jurídica y un aprendizaje significativo que se promueva desde los diferentes escenarios universitarios. En esta ponencia los autores han sistematizado un conjunto de referentes que sustentan la necesidad de una interrelación cultura-educación superior para el logro de las aspiraciones en esta carrera. Se emplearon diversos métodos de la investigación pedagógica para revelar los aportes teóricos.

Palabras clave: educación superior, cultura jurídica, aprendizaje significativo.

\begin{abstract}
The formative process of the students of the career of Right in the extension Riobamba of Uniandes, Ecuador, demands from the articulation of the artificial culture and a significant learning that it is promoted from the different university scenarios. In this report the authors have systematized a group of relating that sustain the necessity of an interrelation culture-superior education for the achievement of the aspirations in this career. Diverse methods of the pedagogic investigation were used to reveal the theoretical contributions.
\end{abstract}

Keywords: superior education, artificial culture, significant learning.

\section{() 2019}

\section{Correspondencia de autor:}

xiglesiasuniandesr@gmail.com xime_cangas@hotmail.com mesimach@hotmail.com

Recibido:

15/Julio/2018

Aceptado:

25/0ctubre/2018

Publicado:

3/Enero/2019 


\section{Introducción}

La segunda década del siglo XXI en Latinoamérica exige de una universidad ajustada a las necesidades del mundo actual, cónsona con las múltiples complejidades de la vida y el entorno socioeconómico cultural y medioambiental en que se desenvuelven, adherida a principios y normas que revelen su pertinencia y le den sentido a su actividad fundamental: formar egresados preparados para las confrontaciones diversas de la cultura en su más amplia expresión. He ahí un reto que requiere de miradas interdisciplinares en que las ciencias sociales, humanísticas y pedagógicas se entrelazan para contribuir al cumplimiento de los objetivos de la Unesco dirigidos al Desarrollo Sostenible en la Agenda 2030 (UNESCO, 2013, 2014, 2015).

Si bien los Objetivos del Milenio trazaron una proyección estratégica en el siglo $X X I$, las aspiraciones planteadas en el 2015, demandan de una educación superior centrada en hacer más efectiva, realista y concreta la producción de saberes en una universidad innovadora, de cara a las potencialidades de lo mejor del ser humano, que muestre su cuidado del medio ambiente, trabaje por la transformación y equidad social, como aspectos esenciales que pueden estar centrados en la interculturalidad (Rodríguez, 2016).

Frente al reconocimiento de la contribución del papel de la ciencia, la tecnología y la innovación para atender los objetivos de la educación de calidad, en especial de la educación superior y la defensa del medio ambiente, con particular énfasis en los ecosistemas marinos y terrestres, la producción de alimentos para eliminar el hambre, es de pertinencia formar una actitud responsable desde la visión ciudadana, lo que hace que el desarrollo de una cultura jurídica se alcance atendiendo a los objetivos de la UNESCO (2015).

En Ecuador, la Constitución de la República en su artículo 350 fija unas perspectivas de largo alcance al desarrollo de la investigación universitaria que potencie en los egresados la formación académica y profesional con visión científica y humanista; la investigación científica y tecnológica; la innovación, promoción, desarrollo y difusión de los saberes y las culturas; la construcción de soluciones para los problemas del país, en relación con los objetivos del régimen de desarrollo. 
Se comparte que la educación superior es un bien público y un imperativo estratégico para todos los niveles de la educación, lo que posee cada vez mayor connotación y exige de la incidencia social como nunca antes en la historia.

Invertir en la educación superior es un reto y una perspectiva ante el desafío de construir sociedades del conocimiento inclusivas y diversas, así como para el avance de la investigación, la innovación y la creatividad (Tunnerman, 2010). Se comparte con Casañas (2015) y Peña (2015) que la investigación en las universidades es agente de cambio, lo que ha llevado a transitar de la I+D hacia la I+D+ i, porque la innovación es esencial en la transformación.

En este contexto formativo y de investigación los autores del presente trabajo insertan sus proyectos investigativos en la carrera de Derecho en la extensión Riobamba, de la Uniandes, Ecuador, encaminados a dar respuesta a las diversas problemáticas en el ámbito de las transformaciones universitarias de esta carrera.

La aplicación de métodos de investigación científica desde la dialéctica materialista pedagógica y etnográfica de interpretación de la información obtenida en documentos normativos, tales como la observación, análisis y síntesis, la interpretación de los resultados de documentos, entrevista, encuestas a directivos, profesores y estudiantes, permitió el seguimiento a los principales resultados de la carrera en el lapso de 2012 a 2016 revelando la necesidad de integrar en el trabajo de los claustros de los diferentes años académicos en la extensión Riobamba, no solo adquisición de los conocimientos de jurisprudencia, sino de saberes pedagógicos y didácticos, que proyecten una mejora en la calidad de los procesos de vinculación e investigación.

En este artículo los autores sistematizaron los referentes teórico-metodológicos comunes a un proceso investigativo en torno a la formación y desarrollo de los estudiantes de la carrera de Derecho en la Uniandes, a partir de la relación cultura y educación superior.

\section{Desarrollo}

En la segunda década del siglo XXI, el examen de diferentes documentos normativos a nivel internacional, denota la significación de la formación universitaria en 
la sociedad actual. UNESCO (2008, 2009, 2013), Díaz (2012), Coll (2013), Hernández y Figuerola (2016) revelan posiciones diversas que apuntan a una nota común, al respecto los últimos autores mencionados comparten que: "una aproximación integral a la formación superior, conjugando los requerimientos de la práctica profesional con el desarrollo de valores éticos y humanísticos" (p.21).

En la formación de los profesionales de la carrera de Derecho en la Uniandes, Ecuador, se toman como referentes esenciales los objetivos expresados en su normatividad así como las aspiraciones sociales y los contextos particulares para los cuales se prepara. Se revela así la necesaria articulación dinámica entre cultura y educación superior.

Es así que se reconocen como antecedentes de esta investigación los aportes relacionados con la formación en la educación universitaria en Uniandes, la cultura jurídica y el aprendizaje significativo en concordancia con el desarrollo de habilidades investigativas, relacionados con las problemáticas, limitaciones, oportunidades y exigencias de la comunidad donde está enclavada la universidad.

La organización de la carrera de manera flexible para promover la ciencia y la interdisciplinariedad al servicio de la sociedad, posibilita establecer una relación dinámica entre las aspiraciones sociales, el proceso de enseñanza-aprendizaje desarrollador y la cultura jurídica como sustento de una pedagogía universitaria radicada en la autonomía universitaria, la libertad de cátedra y la extensión universitaria como expresión de mayor compromiso con los destinos de la sociedad.

El actual sistema de educación superior del Ecuador es concebido con sus procesos de transformaciones internas como un bien público que afectará tarde o temprano a la sociedad en su conjunto, sin importar si es gestionado por actores estatales o particulares, pero sí como parte preponderante de un intelecto colectivo social orientado hacia la sociedad del "Buen Vivir". Al respecto sirven de punto de partida las investigaciones de Quirola (2012), Ramírez $(2012,2013)$ y Larrea $(2014,2015,2016)$.

En el período más reciente (2010 - actualidad) el cambio que comienza a diseñarse en la educación superior ecuatoriana está dirigido a su rescate como bien público, para lo cual pretende colocar al conocimiento, la investigación y la innovación en un lugar central, y busca combinar de la forma más armoniosa posible excelencia (referido 
a cumplimiento de estándares internacionales de calidad en todas las funciones) con pertinencia (por la mayor implicación en el desarrollo social con responsabilidad social).

En la segunda Conferencia Regional de la UNESCO para la Educación Superior (2008) se reveló el necesario compromiso de la Educación Superior con las demandas de la sociedad sobre la base de una capacidad de respuesta reflexiva, rigurosa y crítica, lo que implica a juicio de los autores de este trabajo estar en función del entorno socio-económico, ambiental y el carácter intercultural.

Al respecto, Ramírez $(2013$, p.20) menciona siete pilares para la tercera ola de transformación de la educación superior en el Ecuador: 1) descorporativizar el sistema de educación superior para el bien común; 2) democratizar la educación superior y el conocimiento; 3) generar nuevo conocimiento en el marco de una autonomía universitaria responsable con la sociedad; 4) revalorizar el trabajo docente y de investigación en el sistema de educación superior; 5) endogeneidad regional para la integración latinoamericana y la inserción inteligente a nivel mundial; 6) convergencia hacia arriba, eliminando circuitos diferenciados de calidad; y 7) construir una episteme emancipadora de la sociedad.

Según Ramírez (2012), desde el momento de construcción y promulgación de la Constitución de 2008, comienza la interpelación crítica y propositiva a la universidad ecuatoriana, iniciando un proceso de transformación inédito en la educación superior.

La LOES establece en el inciso (f): "Fomentar y ejecutar programas de investigación de carácter científico, tecnológico y pedagógico que coadyuven al mejoramiento y protección del ambiente y promuevan el desarrollo sustentable nacional" (Presidencia de la República, 2010, p.6).

La UNESCO (2014) plantea que la misión de la Educación Superior es contribuir al desarrollo sostenible y al mejoramiento conjunto de la sociedad, en un mundo globalizado con avances extraordinarios junto con niveles inaceptables de un comportamiento irresponsable respecto del medio ambiente, en un mundo que ya está sintiendo los impactos del cambio climático, es el momento de incrementar los esfuerzos para crear un mundo más justo, seguro y sostenible para todos (UNESCO, 
2014, pp.196-198), de ahí que se asume por los autores de este trabajo la pertinencia de perfeccionar el proceso formativo en la carrera de Derecho desde una educación que ponga énfasis en las necesidades del entorno.

Es de pertinencia en la actividad investigativa que se realiza atender las categorías de formación y desarrollo, como dos procesos que se articulan en la vida universitaria y se concretan en los procesos sustantivos. En la Conferencia Inaugural de Pedagogía 2013, la ministra de Educación de Cuba, se refirió a la necesidad de una educación para la vida, centrada en la atención al ser humano. Al respecto se refirió a cómo deben organizarse los esfuerzos en la región, lo que cobra valor epistemológico en la investigación desarrollada por los autores de este trabajo:

los modos de hacer, pensar y trabajar la cultura de los pueblos, sus valores; se trasmiten de unas generaciones a otras como parte del proceso de socialización en que transcurre la vida, el cual resulta más complejo ahora por los efectos de la globalización neoliberal que trata de imponer formas de actuar y modos de vida únicos. (Velázquez, 2012, p.6)

La educación científica del siglo XXI forma al profesional con competencias claves para su desarrollo integral, la educación ecuatoriana tiende a la preparación de un profesional altruista, autónomo y creativo, por ello se considera que la tarea de formar e informar a los juristas debe ser una preocupación de todos, trata de formar un profesional vinculado a la problemática social, que comprenda la naturaleza real del Derecho como ciencia, donde se combinen propósitos prácticos y profesionales con otros estrictamente teóricos o científicos con la aplicación de las TIC.

Se comparte con Casañas (2016) que "el siglo XXI necesita un individuo con una filosofía diferente, ambientalista, y humanista por esencia, una ética basada en la equidad y la solidaridad, donde el respeto al otro, cualquiera que sea su cultura 0 raza, constituya un principio inviolable" (p.15).

A juicio de estos los del artículo esta idea es clave en la formación del jurista que debe integrar un pensamiento crítico, sustentado en la cultura. Al respecto se comparte el concepto formación que plantea la Organización de las Naciones Unidas para la Educación, la Ciencia y la Cultura (UNESCO): “[...] implica la adquisición de 
conocimientos, actitudes, habilidades y conductas íntimamente asociados al campo profesional" (Alba, 2012).

La Universidad Regional Autónoma de los Andes (Uniandes) se encuentra en la ciudad de Ambato y sus extensiones a nivel nacional La carrera de Derecho funciona desde el año 1998.

La carrera de Derecho en la Facultad de Jurisprudencia de la Universidad Regional Autónoma de los Andes (Uniandes) se sustenta en un enfoque en la formación humanista, crítica, emprendedora e innovadora, con miras a la construcción de una sociedad justa, libre y democrática. Es de suma importancia que sus estudiantes desarrollen conocimientos, habilidades, valores, técnicas en determinadas asignaturas a efectos de que se puedan involucrar de manera directa con la ciencia y la tecnología en la sociedad para la cual se están preparando.

Se forma un profesional vinculado a la problemática social para comprender la naturaleza real del Derecho como ciencia, valorado como un fenómeno en sí mismo, donde se convinen procesos prácticos y profesionales con otros estrictamente teóricos o científicos.

Entre las competencias claves a desarrollar en los estudiantes se reconocen:

- La eficiencia en la organización del trabajo.

- La responsabilidad en el trabajo.

- La coherencia en el trabajo de equipo.

- La autonomía e interrelación.

- La posibilidad de garantizar efectivas relaciones interpersonales.

- La generación de iniciativas, innovación y creatividad.

- La toma de decisiones oportunas y acertadas.

- La flexibilidad y apertura al cambio.

- La buena comunicación y empatía con los que los rodean.

- La sencillez, honestidad y modestia en la actuación para con los otros.

De ahí que es importante la preparación científica, el desarrollo de habilidades en la actividad científica, el desarrollo de una cultura jurídica en un proceso de enseñan- 
za-aprendizaje en que se articulen de manera armónica y significativa los componentes prácticos y teóricos de la jurisprudencia.

Los aportes de Coll (2013) acerca de la ecología del aprendizaje revelan las necesarias adecuaciones y la posibilidad de la creatividad en la actividad formativa de la carrera de Derecho en Uniandes, en un entorno de aprendizaje apoyado en la actividad científica investigativa.

El aprendizaje significativo y el aprendizaje autónomo son de gran pertinencia, si se logra por parte de los docentes potenciar los conocimientos en función de las tradiciones sociocomunitarias, activar la conciencia acerca del proceso de cambio en la educación contemporánea, que promueva un aprendizaje que le sirva para la vida y el desenvolvimiento profesional, que le permita desarrollar competencias básicas en su profesión, dar paso a las realidades más alejadas de los centros de poder y de la educación en la cual las habilidades y destrezas cumplan un papel fundamental en su desempeño profesional así como el saber convivir y compartir con los demás.

Los aprendizajes autónomos (Trejo, et al., 2017) son importantes para lograr una mayor seguridad, motivación, actitud consciente hacia lo que se aprende, y en ello tienen un valor indispensable las técnicas y procedimientos didácticos que el docente emplee como mediador y además como vía para la independencia y la creatividad en la solución de los problemas profesionales, de ahí que uno de los elementos fundamentales va a estar vinculado con la actividad científico-investigativa.

De estos aspectos, los autores de este artículo convergen en señalar como aspectos sistematizados los siguientes:

- La formación de los estudiantes de la carrera de Derecho en Uniandes, extensión Riobamba, es un proceso en el cual se involucran diferentes agentes y actores, pero son los propios estudiantes lo que mejor y mayor énfasis pueden hacer en la medida que logran confianza, autoestima, sistematicidad en sus relaciones e interacción con la cultura de la profesión, en la significatividad de sus aprendizajes.

- El contenido de la formación referido a la cultura que debe alcanzar un profesional, abarca no solo los conocimientos científicos y tecnológicos que respondan a una rama y objeto del saber, sino a la cultura como 
resultado de un tipo específico de educación científico-tecnológica. Esta última es entendida como: el proceso continuo de adquisición de conocimientos teóricos y prácticos y de formación de valores en relación con la práctica tecno-científica, propiciadora de una actitud crítica de los aspectos contradictorios presentes en las relaciones entre la actividad científico-tecnológica y las otras formas de actividad social.

- La cultura jurídica se ha definido como el sistema de valores socialmente dominantes, sobre lo justo y lo injusto de una determinada acción u omisión, resultado no solo de la costumbre y del grado de instrucción, sino además de los intereses económicos y sociales prevalecientes en ese momento.

- La cultura jurídica deviene un pilar fundamental porque inserta los valores que se deben interconectar en los modos de actuación del jurista. Una cultura jurídica a tono con las necesidades y exigencias del contexto en que vive, enriquece la educación superior por los aportes que realiza en las posibilidades de aplicación del marco constitucional.

- Desde lo académico se debe contribuir a un proceso de enseñanzaaprendizaje en el cual se alcance una sólida e independiente adquisición de nuevos saberes por parte de los estudiantes, con un pensamiento crítico y creador hasta llegar a una transformación renovadora mediante la práctica investigativa.

Al respecto, sirve de sustento lo expresado en los documentos normativos

El maestro es el orientador, guía, induce al estudiante a resolver problemas en forma activa. Una de las ventajas de esta teoría, es el incremento en el desarrollo intelectual y a la vez, retiene el conocimiento en la memoria de forma organizada, lo que permite recordar con facilidad, expresa que aprender es un redescubrir, un reinventar entendiendo que el estudiante va construyendo su estructura cognoscitiva de una manera personal, autónoma, él es el protagonista de su propio aprendizaje. (Universidad Nacional de Chimborazo - UNACH, 2014) 
En el contexto educativo de la carrera de Derecho es importante y significativo saber dónde se aprende, cuándo, con quién y de quién, cómo, qué e incluso para qué aprende. Los entornos áulicos son disputados hoy como escenarios de aprendizaje porque alcanza notoriedad el uso de los dispositivos móviles, y otras alternativas para apropiarse de los contenidos social y culturalmente relevantes en cada una de las materias, lo que lleva a los docentes a replantearse los métodos y procedimientos a emplear. Cada vez hay un amplio abanico de contextos de actividad y en interacción con una diversidad de actores y agentes educativos en los cuales ocurre el aprendizaje, que lo hacen ser significativo y vivencial.

En este proceso, el uso de las TIC, particularmente los dispositivos móviles con conexión inalámbrica a Internet, ofrecen facilidades para la conexión de aprendizajes y experiencias de aprendizaje que ocurren fuera de los contextos institucionales y que tienen una implicación para la producción de nuevos aprendizajes.

Se comparte con Morin y Delgado (2017), el desafío planetario que la universidad con su sinergia debe asumir:

Es indudable que el desarrollo de la investigación científica, desde la modernidad, y la acelerada instrumentación práctica del conocimiento en las tecnologías, desde el inicio de la era industrial, nos han colocado en una posición privilegiada como poseedores de saberes que modifican el mundo. (p.18)

La sociedad ecuatoriana actual demanda de una universidad que integre la cultura que entronque lo mejor de la investigación y el desarrollo de cara a la innovación para una puesta en práctica e implementación de los mejores resultados, como un componente esencial de la calidad, pertinencia y viabilidad de la universidad.

La formación de los estudiantes de Derecho de Uniandes, desde una concepción integradora y sistémica, articula la tradición cultural y las nuevas técnicas de enseñanza que den posibilidades de transformar los contextos sociocomunitarios de los más recónditos lugares del Chimborazo con acciones que cuiden de los valores ciudadanos.

El desarrollo de investigaciones en dependencia de los intereses de las comunidades en que se ubica la universidad, de los grupos, urbanidades y territorios para 
los cuales produce sus saberes, debe estar dirigido a resolver los problemas de la sociedad y la comunidad en su conjunto y en sus particularidades.

La relación de educación y cultura en la formación de los estudiantes de la carrera de Derecho halla un sustento pertinente en la siguiente idea:

Es clave que la educación como proceso formativo gravite en torno a valores humanos que impulsen una nueva ética de la investigación donde se desmantele el discurso tecnocientífico plagado de peligros y se promueva la responsabilidad para con el planeta. (Parada, 2013, p.196)

En el contexto de la carrera de Derecho en la Uniandes se establecen de manera precisa las formas de participación de los estudiantes en las acciones investigativas en torno a problemáticas de la región de Riobamba, a las cuales se le puede dar respuesta mediante los distintos tipos y niveles de investigación, con proyectos multidisciplinarios que involucren y sensibilicen a la institución; la investigación con base en proyectos, formativa o para el aprendizaje que se ejecuta con proyectos integradores como aplicación de los conocimientos científicos y las habilidades investigativas adquiridas.

\section{Conclusiones}

La perspectiva pedagógica y didáctica en la educación superior constituye una exigencia en el proceso formativo de la carrera de Derecho en Uniandes, extensión Riobamba, que se sustenta en los referentes teóricos y metodológicos desde lo jurídico. En la formación de este profesional se abren nuevos desafíos en la época actual, frente a la necesaria sensibilización de la humanidad por la especie humana, la universidad debe formar juristas en un aprendizaje significativo, con un desarrollo de habilidades científico-investigativas que amplíe su cultura de la profesión y posibilite su actitud transformadora.

\section{Referencias bibliográficas}

Alba, 0. (2012). La formación de profesionales emprendedores: necesidad socioeconómica, retos y vías en el contexto de la Educación Técnica y Profesional cubana. (pp.1-57). Curso Pre-evento. Actas del VIII Taller Nacional de la ETP y VI Coloquio 
de Formación Laboral (Santiago de Cuba), Cuba. [CD-ROM] Santiago de Cuba: UCP Frank País García.

Casañas, M. (2015). La investigación en la educación superior en América Latina. Revista Amauta, 13(25). enero-junio, 67-78. Universidad del Atlántico, Barranquilla, Colombia.

Casañas, M. (2016). Introducción a La formación de valores en nuestra América. Colección Educación Emancipadora. Caracas, Venezuela: Imprenta Universidad Bolivariana de Venezuela.

Caveda, D. A. (2010). La formación investigativa en la carrera de derecho: los estudios jurídicos en la Universidad de Pinar del Río. Revista Odiseo, revista electrónica de pedagogía, 8(15). Recuperado de: http://www.odiseo.com.mx/2010/8-15/ caveda-estudios-juridicos.html

Coll, C. (2013). Aprendizaje y educación en la sociedad digital. Barcelona: Universitat de Barcelona.

Díaz, T. (2014). Fundamentos pedagógicos y didácticos de la Educación Superior. Universidad 2014. Curso Corto 15. La Habana: Editorial Universitaria.

Hernández, H. \& Figuerola, M. (2016). Miradas pedagógicas a la formación universitaria en documentos político-académicos. Reflexiones sobre la universidad en Cuba. En: T. Ortiz \& T. Sanz (Coord.) Visión pedagógica de la formación universitaria actual (pp.17-42) La Habana: Editorial UH.

Larrea, E. \& Granados, V. (2016). El Sistema de Educación Superior para la Sociedad del Buen Vivir basada en el conocimiento: el caso ecuatoriano. Guayaquil, Ecuador: Universidad Católica de Santiago de Guayaquil.

Larrea, E. (2014). El currículo de la Educación Superior desde la complejidad sistémica. Algunas consideraciones para orientar el proceso de construcción del nuevo modelo de formación universitaria. Guayaquil, Ecuador: CES. Recuperado de http://www.ces.gob.ec

Larrea, E. (2015). El abordaje de la formación profesional a partir de la configuración de constructos teórico-metodológicos, sistémicos y pertinentes. Guayaquil, Ecuador: CES.

López, J. A. \& Sánchez, J. M. (eds.) (2001). Ciencia, tecnología, sociedad y cultura en el cambio de siglo. Madrid: Biblioteca Nueva. Organización de Estados Iberoamericanos. 
Morin, E. \& Delgado, C. J. (2017). Reinventar la educación. Abrir los caminos hacia la metamorfosis. La Habana: Universidad de La Habana.

Núñez, J. \& López, J. A. (2001). Innovación tecnológica, innovación social y Estudios CTS en Cuba. En: A. Ibarra \& J. A. Cerezo (eds.) Desafíos y tensiones actuales en Ciencia, Tecnología y Sociedad. Madrid: Biblioteca Nueva. Organización de Estados Iberoamericanos.

Parada, J.A. (2013). Retos a la educación contemporánea en la era de la tecnociencia. Revista Amauta, 11(21), 181-202. Universidad del Atlántico, Barranquilla, Colombia.

Peña, C. A. (2015). La importancia de la investigación en la universidad: una reivindicación del sapere aude kantiano. Revista Amauta, 13(25). Universidad del Atlántico, Barranquilla, Colombia.

Presidencia de la República del Ecuador (2008). Constitución de la República del Ecuador. Editada por LEXIS S. A. Documento en formato digital.

Presidencia de la República del Ecuador (2010). Ley Orgánica de Educación Superior. Editada por LEXIS S. A. Documento en formato digital.

Quirola, D. (2012). La universidad ecuatoriana en la transición hacia la sociedad del Buen Vivir basada en el bioconocimiento. En: J. Ramírez, ed. Transformar la universidad para transformar la sociedad. Segunda edición. Quito, Ecuador: SENESCYT.

Ramírez, J. (2012). Transformar la universidad para transformar la sociedad. Segunda edición. Quito, Ecuador: SENESCYT.

Ramírez, J. (2013). Tercera ola de transformación de la Educación Superior en Ecuador. Hacia la constitucionalización de la sociedad del Buen Vivir. Quito, Ecuador: SENESCYT.

Rodríguez, J. L. (2016). Ciencias de la Educación, interculturalidad y formación humanística en la universidad cubana, caribeña y latinoamericana Contemporánea. Quito: UNIANDES / Editorial Jurídica del Ecuador.

Trejo, M. L et al. (2017). Creencias de las capacidades para ejercer la autonomía en un ambiente de aprendizaje. El caso de la Facultad de Lenguas, Tuxtla de la UNACH. En: M. Chang, M. L. Trejo \& M. E. Llaven, coord. La autonomía en ambientes de aprendizaje. Ibiza, España: Fondo Editorial Universitario.

Tunnerman, C. (sept/diciembre 2010). Las conferencias regionales y mundiales sobre educación superior de la UNESCO y su impacto en la educación superior de América Latina. Revista Universidades, LX(47). 
UNESCO (2008). Declaración Final de la Conferencia Regional de Educación Superior en América Latina y el Caribe. En: Ciencia, Tecnología, Sociedad e Innovación para el Desarrollo Sostenible. OIE. Recuperado de http://www.oei.es/historico/ salactsi/cres.htm 2 de abril de 2018.

UNESCO (2009). Nueva dinámica de la educación superior y la búsqueda del cambio social y el desarrollo. Conferencia mundial de Educación Superior. Comunicado oficial. París.

UNESCO (2013). CONFERENCIA GENERAL 37a reunión, París 2013. Conferencia 37, propuesta de programa de Acción Mundial de Educación para el Desarrollo Sostenible (EDS) Como seguimiento del decenio de las Naciones Unidas de la Educación para el Desarrollo Sostenible después de 2014. (pp.37-57). París: UNESCO.

UNESCO (2014). Propuesta de Programa de Acción Mundial de Educación para el Desarrollo Sostenible (EDS) como seguimiento del decenio de las Naciones Unidas de la Educación para el Desarrollo Sostenible después de 2014. Recuperado de: http://mercosurambiental.net/MD_upload/Archivos/1/File/biblioteca/pdf/suelo/ conv-spa.pdf, 1994.

Universidad Nacional de Chimborazo (2014). Modelo Educativo Pedagógico Didáctico. Riobamba.

Velázquez, E. E. (enero, 2013). Educación para la vida: un desafío permanente a los sistemas educacionales de América Latina y el Caribe. En: Memorias del Congreso Pedagogía 2013. Conferencia Inaugural. La Habana: MINED. 